Check for updates

Cite this: Analyst, 2020, 145, 1389

Received 3rd December 2019,

Accepted 6th January 2020

DOI: $10.1039 / c 9 a n 02436 k$

rsc.li/analyst

\section{A high-selectivity fluorescent probe for hypoxia imaging in cells and a tumor-bearing mouse model $\dagger$}

\author{
Yue Wang, ${ }^{a, b}$ Xiaoyue Han, ${ }^{a}$ Xia Zhang, ${ }^{a, b}$ Li Zhang $^{a, b}$ and Lingxin Chen (D) *a,c,d
}

Nitroreductase (NTR) with a high expression level in tumors has been considered as a biomarker of highly aggressive hypoxia tumors. Thus, it is important to develop powerful tools for tumor hypoxia detection. Here, we developed a two-photon fluorescent probe hTP-NNO 2 for NTR detection. The probe with onestep synthesis exhibited high yield. hTP- $\mathrm{NNO}_{2}$ showed high selectivity and sensitivity for NTR and the detection limit was as low as $43 \mathrm{ng} \mathrm{mL} \mathrm{m}^{-1}$. hTP- $\mathrm{NNO}_{2}$ also showed low cytotoxicity and high stability, indicating that hTP-NNO is suitable for NTR detection in real-time and in situ under physiological conditions. hTP- $\mathrm{NNO}_{2}$ was used for NTR imaging in hypoxia cells and the fluorescence intensity of hTP-NNO $\mathrm{N}_{2}$ increased with decreasing oxygen concentration. Benefiting from the advantages of twophoton fluorescent probes, we performed NTR detection in deep brain tissue with an imaging depth of up to $100 \mu \mathrm{m}$. hTP-NNO 2 was further successfully applied for NTR detection in zebrafish and tumors. These results indicated that we developed a promising fluorescence imaging tool for NTR detection in vitro and in vivo.

\section{Introduction}

Hypoxia is a pathological oxygen deficiency state in organisms, where the oxygen concentration is in the range from $4 \%$ to $0 \% .{ }^{1,2}$ Hypoxia is conducive to the progression and metastasis of malignant tumors. ${ }^{3-5}$ Hypoxia can also increase the possibilities of gene mutations and lead to overexpression of many tumor-associated factors, particularly hypoxia-inducible factor 1 alpha (HIF-1 $\alpha$ ), and of many HIF-mediated genes. ${ }^{6,7}$ In addition, hypoxia is detrimental to the tumor prognosis because it leads to resistance to standard therapies and it promotes a more malignant phenotype..$^{9-11}$ Lots of previous studies have shown that the hypoxic state of tumors is closely related to the process of tumor progression. ${ }^{5,8}$ Therefore, effective tools for tumor hypoxia detection are needed urgently for evaluating tumor progression. Nitroreductase (NTR) is an endogenous enzyme that is overexpressed in a hypoxia micro-

${ }^{a}$ CAS Key Laboratory of Coastal Environmental Processes and Ecological Remediation, Yantai Institute of Coastal Zone Research, Chinese Academy of Sciences, Yantai 264003, China. E-mail: lxchen@yic.ac.cn

${ }^{b}$ University of Chinese Academy of Sciences, Beijing 100049, China

${ }^{c}$ School of Pharmacy, Binzhou Medical University, Yantai 264003, China

${ }^{d}$ Center for Ocean Mega-Science, Chinese Academy of Sciences, Qingdao 266071, China

$\dagger$ Electronic supplementary information (ESI) available. See DOI: 10.1039/ c9an02436k environment especially in tumors, and the expression level of NTR is closely associated with the tumor progression and the hypoxia degree. ${ }^{1,12}$ We can assess the hypoxia level by measuring the concentration fluctuations of NTR. ${ }^{13-15}$ Therefore, effective tools for NTR detection are needed urgently for evaluating tumor progression and diagnosis.

Traditional methods for hypoxia detection include electron paramagnetic resonance imaging (EPRI), $\mathrm{pO}_{2}$ electrodes, positron emission tomography/computed tomography (PET/CT) and so on. ${ }^{17-19}$ However, these methods cannot achieve realtime and in situ imaging for NTR detection due to complex sample pretreatment, destruction of tissues or cells. Fluorescence imaging technology has been employed as a powerful tool for the detection of active species due to its advantages including rapid response, superior selectivity, outstanding spatial and temporal resolution, less invasiveness, and real-time and in situ detection..$^{20,21}$ So far, many fluorescent probes have been developed for NTR detection, and most of them are one-photon fluorescent probes. ${ }^{22-25}$ Twophoton fluorescent probes use two near-infrared photons as an excitation source, and have obvious advantages over onephoton fluorescent probes, such as deeper tissue imaging depth, higher spatial and temporal resolution and longer observation time. ${ }^{26,27}$ The developed two-photon fluorescent probes for NTR detection have short fluorescence emission wavelengths, which causes the interference due to shallow penetration depth and autofluorescence of intrinsic bio- 
molecules. ${ }^{28}$ Thus, there is an urgent need to develop simple and convenient two-photon fluorescent probes with near-infrared fluorescence for NTR quick detection.

Here, we successfully designed and synthesized a twophoton fluorescent probe, hTP- $\mathrm{NNO}_{2}$, for NTR detection with near-infrared fluorescence emission at $675 \mathrm{~nm}$. We selected $p$-nitrobenzene as the response site and the synthesis methods of the probe based on a two-photon fluorophore TP-NH were simple and convenient. hTP- $\mathrm{NNO}_{2}$ was successfully used for NTR detection in vitro and in living cells at different oxygen concentrations. We also performed NTR detection using a twophoton laser confocal microscope in deep brain tissue. We could capture cellular activities at each layer throughout the tissue, and the imaging depth was up to $100 \mu \mathrm{m}$. Probe hTP-NNO $\mathrm{N}_{2}$ was further employed for NTR detection in zebrafish and solid tumors.

\section{Experimental section}

\subsection{Synthesis method of compound hTP-NNO}

Compound TP-NH (0.0355 g, $0.1 \mathrm{mmol})$ and $\mathrm{Cs}_{2} \mathrm{CO}_{3}(0.16 \mathrm{~g}$, $0.5 \mathrm{mmol}$ ) were dissolved in DMF $(30 \mathrm{~mL})$ for $30 \mathrm{~min}$ at $0{ }^{\circ} \mathrm{C}$. Then 4-nitrobenzyl bromide $(0.0432 \mathrm{~g}, 0.2 \mathrm{mmol})$ was added and the solution was stirred overnight. After concentration, the obtained crude product was purified by silica column chromatography (200-300 mesh) to afford the final product $(0.0387$, 78.9\%). ${ }^{1} \mathrm{H}$ NMR (500 MHz, DMSO-D6) $\delta$ (ppm): 1.635-1.670 $(\mathrm{t}, 3 \mathrm{H}), 1.841-1.885(\mathrm{~s}, 6 \mathrm{H}), 3.229-3.311(\mathrm{~s}, 3 \mathrm{H}), 4.423-4.529$ $(\mathrm{s}, 1 \mathrm{H}), 4.772-4.885(\mathrm{~s}, 2 \mathrm{H}), 4.901-5.038(\mathrm{q}, 2 \mathrm{H}), 6.821-6.934$ (s, 1H), 7.400-7.435 (d, 2H), 7.537-7.589 (m, 4H), 7.645-7.694 (d, 1H), 7.768-7.830 (d, 1H), 8.003-8.052 (d, 1H), 8.055-8.110 (d, 1H), 8.139-8.186 (d, 1H), 8.188-8.253 (d, 2H), 8.256-8.319 (d, 1H). LC-MS (API-ES): $m / z \mathrm{C}_{32} \mathrm{H}_{32} \mathrm{~N}_{3} \mathrm{O}_{2}{ }^{+}$calcd 490.61, found $\left[\mathrm{M}^{+}\right]$490.46.

\subsection{Cell culture}

Human lung carcinoma (A549) cells and human neuroblastoma (SH-SY5Y) cells were purchased from the Committee on Type Culture Collection of Chinese Academy of Sciences (Shanghai, China). A549 cells were cultured with DMEM supplemented with 10\% FBS. SH-SY5Y cells were cultured with RPMI-1640 supplemented with 10\% FBS. All cells were incubated at $37{ }^{\circ} \mathrm{C}$ under a humidified atmosphere containing $5 \%$ $\mathrm{CO}_{2}$. The cells were passaged by scraping and seeding on $20 \mathrm{~mm}$ Petri dishes according to the manufacturer's instructions. A multi gas incubator (Sanyo) was used for producing 1-21\% $\mathrm{O}_{2}$ concentration by means of $\mathrm{N}_{2}$ substitution. $0.1 \% \mathrm{O}_{2}$ concentration was generated using an AnaeroPack ${ }^{\mathrm{TM}}$ (Mitsubishi Gas Chemical Company, Co. Inc., Japan).

\subsection{Establishment of A549 transplanted tumor nude mice}

5-Week-old specific pathogen-free nude mice were provided by Changzhou Cavens Lab Animal Co. Ltd. The mice were housed in individual ventilated cages, and given free access to a SPF laboratory diet and water. Mice were group-housed in a $12: 12$ light-dark cycle. $2 \times 10^{6}$ cells were suspended in media and injected subcutaneously into nude mice. A549 xenografts were established in nude mice until the tumor volumes typically reached about $200 \mathrm{~mm}^{3}$. All experiments were performed in accordance with the guidelines established by the Committee of Animal Research Policy of Binzhou Medical University.

\section{Results and discussion}

\subsection{Design and synthesis of hTP- $\mathrm{NNO}_{2}$}

Traditional one-photon fluorescent probes are limited by shallow penetration depth, photobleaching and interferences from environmental factors. Thus, we are committed to design a two-photon fluorescent probe for NTR detection. A series of nitroaryl compounds can be reduced to aminoaryl derivatives by NTR with a cofactor, reduced nicotinamide adenine dinucleotide $(\mathrm{NADH})$ or nicotinamide adenine dinucleotide phosphate (NADPH). ${ }^{16}$ We selected $p$-nitrobenzene as a response unit and the two-photon fluorophore TP-NH with near-infrared (NIR) fluorescence emission at $675 \mathrm{~nm}$ was used as a chromophore for NTR detection (Scheme 1). As expected, we observed weak fluorescence from the probe hTP- $\mathrm{NNO}_{2}$ because the response unit $p$-nitrobenzene can quench the fluorescence of hTP-NNO ${ }_{2}$. After reaction with NTR using NADH as a cofactor, the response unit $p$-nitrobenzene changed to an aminobenzene group and hTP-NNO${ }_{2}$ converted to TP-NH through a 1,6rearrangement elimination reaction to emit a fluorescence signal at $675 \mathrm{~nm}$. Compared to the developed two-photon fluorescent probe for NTR detection, ${ }^{3,8,15,30,31}$ the probe hTP-NNO with simple synthesis steps showed high yields $(78.9 \%)$. In addition, the probe hTP- $\mathrm{NNO}_{2}$ with NIR fluorescence wavelength at $675 \mathrm{~nm}$ had a deeper tissue imaging depth and showed less endogenous autofluorescence with an imaging depth of up to $100 \mu \mathrm{m}$. The detailed synthesis route to hTP- $\mathrm{NNO}_{2}$ is shown in Scheme $\mathrm{S} 1 . \dagger$

\subsection{Spectral properties of the probe hTP-NNO ${ }_{2}$ towards NTR}

The spectral properties of the probe hTP- $\mathrm{NNO}_{2}$ towards NTR were determined under simulated physiological conditions

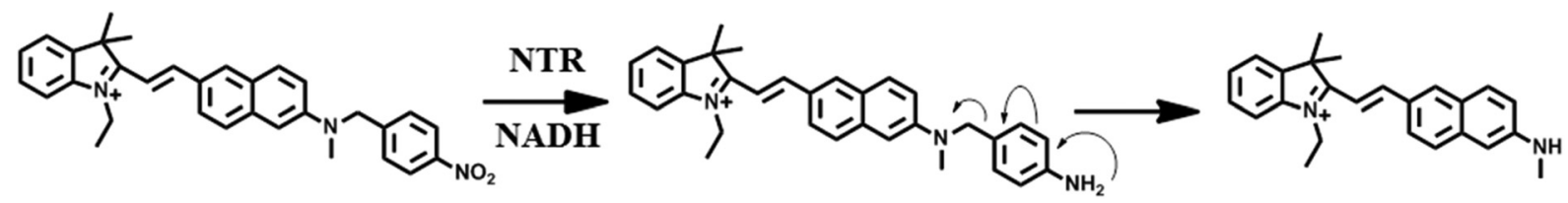

Scheme 1 Illustration of hTP-NNO 2 structure and the reaction mechanism of NTR detection. 

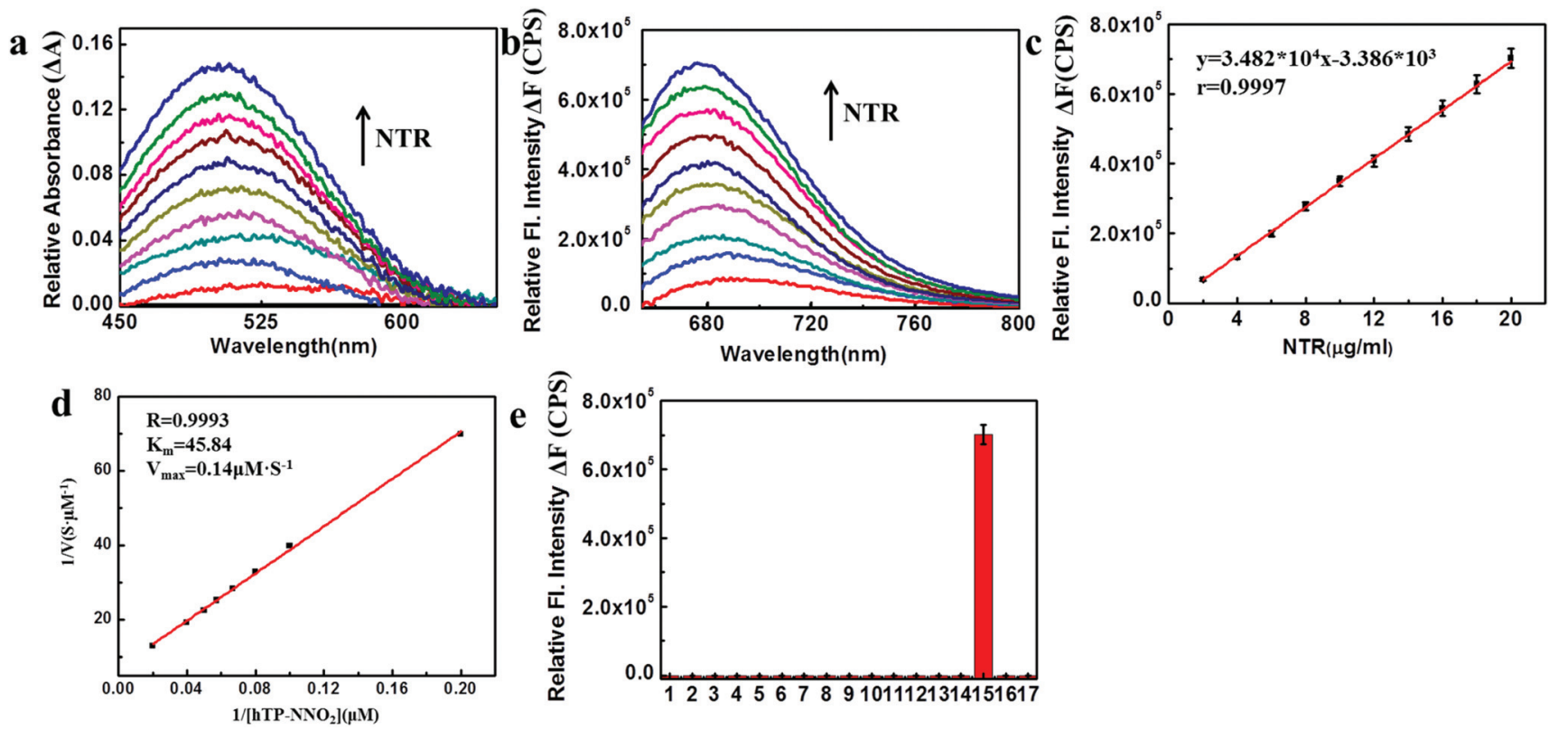

Fig. 1 Spectral properties of hTP-NNO 2 towards NTR. (a) Dose-dependent relative UV-vis absorbance spectra of hTP-NNO $(10 \mu M)$ towards NTR $\left(0-20 \mu \mathrm{g} \mathrm{ml}^{-1}\right)$. (b) Dose-dependent relative fluorescence spectra of hTP-NNO $2(10 \mu \mathrm{M})$ towards NTR $\left(0-20 \mu \mathrm{g} \mathrm{mL} \mathrm{m}^{-1}\right)$. Spectral data were recorded after 10 min of incubation with different concentrations of NTR in the presence of NADH (HEPES pH 7.4, 10 mM). (c) The linear relationship between the relative fluorescence intensities and the NTR concentrations. The experiments were repeated three times and the data are shown as mean \pm S.D. (d) The Lineweaver-Burk plot of the enzyme-catalyzed reaction. hTP-NNO $2(5,10,12.5,15,17.5,20,25$, and $50 \mu \mathrm{M})$. (e) Selectivity detection of hTP- $\mathrm{NNO}_{2}(10 \mu \mathrm{M})$ towards various interfering species. 1, blank; 2, $\mathrm{Fe}^{3+}(1 \mathrm{mM}) ; 3, \mathrm{Ca}^{2+}(1 \mathrm{mM}) ; 4, \operatorname{vitamin~C}(1 \mathrm{mM}) ; 5, \mathrm{H} \mathrm{O}_{2}(100 \mu \mathrm{M}) ; 6, \mathrm{NaClO}$ $(1 \mathrm{mM}) ; 7$, homocysteine $(100 \mu \mathrm{M}) ; 8$, tyrosine $(100 \mu \mathrm{M}) ; 9$, dithiothreitol $(1 \mathrm{mM}) ; 10, \mathrm{H}_{2} \mathrm{~S}_{n}(1 \mathrm{mM}) ; 11$, glutathione $(10 \mathrm{mM}) ; 12$, serine $(100 \mu \mathrm{M}) ; 13$, cysteine $(100 \mu \mathrm{M}) ; 14, \mathrm{NADH}(0.5 \mathrm{mM}) ; 15, \mathrm{NADH}(0.5 \mathrm{mM})+\mathrm{NTR}\left(20 \mu \mathrm{g} \mathrm{m}^{-1}\right) ; 16$, glutathione peroxidase (10 U); and 17, matrix metalloprotein-9 $\left(100 \mathrm{U} \mathrm{L}^{-1}\right)$.

(10 mM HEPES, pH 7.4). The probe showed a sensitive spectral response towards NTR. The relative absorbance spectra of hTP-NNO $\mathrm{N}_{2}$ increased with increasing NTR concentrations, and the absorption peak was centered at $515 \mathrm{~nm}$ (Fig. 1a). Then the relative fluorescence spectra were obtained at different concentrations of NTR $\left(0-20 \mu \mathrm{g} \mathrm{mL}^{-1}\right)$ with $0.5 \mathrm{mM} \mathrm{NADH}$ as a cofactor. The fluorescence intensity of hTP- $\mathrm{NNO}_{2}$ increased with increasing NTR concentration, indicating that the response group in hTP- $\mathrm{NNO}_{2}$ was cleaved and TP-NH was released (Fig. 1b). There was a good linear relationship between the relative fluorescence intensity and NTR concentrations $(0-20 \mu \mathrm{g}$ $\left.\mathrm{mL}^{-1}\right)$. The linear regression equation is $F_{675 \mathrm{~nm}}=3.482 \times 10^{4} \times$ [NTR] $-3.386 \times 10^{3}$ with $r=0.9997$ (Fig. 1c). The detection limit was determined to be $43 \mathrm{ng} \mathrm{mL}^{-1}(3 \sigma / \kappa)$, where $\sigma$ is the standard deviation of blank measurement and $k$ is the slope of the regression equation. These results proved that the probe exhibited a good spectral response towards NTR.

Reaction kinetics was an important parameter for a new fluorescent probe. The curve of the reaction kinetics of hTP-NNO $\mathrm{N}_{2}$ towards NTR was obtained using NADH as a cofactor. We found that the reaction rate increased gradually with the increasing concentration of hTP- $\mathrm{NNO}_{2}$, as shown in Fig. 1d. A Lineweaver-Burk double-reciprocal plot of $1 / V$ ( $V$ is the initial reaction rate) versus the reciprocal of the probe hTP-NNO $\mathrm{N}_{2}$ concentration was used to describe the reaction kinetics of hTP- $\mathrm{NNO}_{2}$, and the kinetic equation is $V=$
$V_{\max }[$ probe $] /\left(K_{\mathrm{m}}+\right.$ [probe] $)$. After calculation, $K_{\mathrm{m}}$ and $V_{\max }$ were found to be $45.84 \mu \mathrm{M}$ and $0.14 \mu \mathrm{M} \mathrm{s}^{-1}$, respectively. These results indicated that hTP- $\mathrm{NNO}_{2}$ possesses high sensitivity towards NTR.

The selectivity of probes was another important parameter for NTR detection. To assess the selectivity of hTP- $\mathrm{NNO}_{2}$ towards NTR, hTP-NNO $\mathrm{N}_{2}$ was tested in a wide variety of interfering species. The concentrations of other interfering substances were higher than their original level in the cells. As shown in Fig. 1e, physiologically relevant ions such as $\mathrm{Fe}^{3+}, \mathrm{Ca}^{2+}, \mathrm{Na}^{+}, \mathrm{K}^{+}$ and $\mathrm{Mg}^{2+}$ cannot cause fluorescence changes. Other active species such as vitamin $\mathrm{C}, \mathrm{H}_{2} \mathrm{O}_{2}, \mathrm{H}_{2} \mathrm{~S}_{n}$, glutathione and cysteine also cannot interfere with the NTR detection. hTP- $\mathrm{NNO}_{2}$ showed apparent fluorescence changes after being reacted with NTR in the presence of NADH. Thus, the probe hTP-NNO showed high selectivity and sensitivity for NTR detection. These results demonstrated that hTP- $\mathrm{NNO}_{2}$ can be used for NTR detection in a complex biological environment and that hTP-NNO $\mathrm{N}_{2}$ is a promising tool for NTR detection in vivo.

\subsection{NTR detection in living cells}

Encouraged by the good spectral properties of the probe hTP-NNO $\mathrm{N}_{2}$ towards NTR in a simulated buffer system, we further explored NTR detection in living cells. We firstly performed MTT assays to evaluate the cytotoxicity of hTP- $\mathrm{NNO}_{2}$. As shown in Fig. S2, $\uparrow$ A549 and SH-SY5Y cells were incubated 
with different concentrations of the probe hTP- $\mathrm{NNO}_{2}$ $(0-70 \mu \mathrm{M})$. The cells showed high viability, indicating that the probe had low cytotoxicity. Next, we detected the NTR concentration fluctuations in the cells at different oxygen concentrations. The cells were incubated with different oxygen concentrations $(0.1 \%, 1 \%, 3 \%, 5 \%, 10 \%$ and $21 \%)$ for $6 \mathrm{~h}$, and then the cells were incubated with $10 \mu \mathrm{M}$ probe hTP- $\mathrm{NNO}_{2}$ for $25 \mathrm{~min}$ at $37^{\circ} \mathrm{C}$ before imaging. As shown in Fig. 2a, our probe showed very weak fluorescence signals at a normal oxygen concentration $(21 \%)$. The fluorescence signals increased gradually with decreasing oxygen concentrations. hTP- $\mathrm{NNO}_{2}$ showed stronger fluorescence signals in hypoxia cells especially in the cells that were incubated with $0.1 \%$ oxygen concentration. The corresponding quantitative analysis of fluorescence of Fig. 2a is shown in Fig. 2c. Flow cytometry analysis has been considered as a high-throughput assay technology for the analysis of a large number of samples. Therefore, we used flow cytometry analysis for NTR detection to confirm the results obtained using a confocal microscope, and these results are shown in Fig. 2b. The fluorescence intensity, as shown in Fig. 2b, was
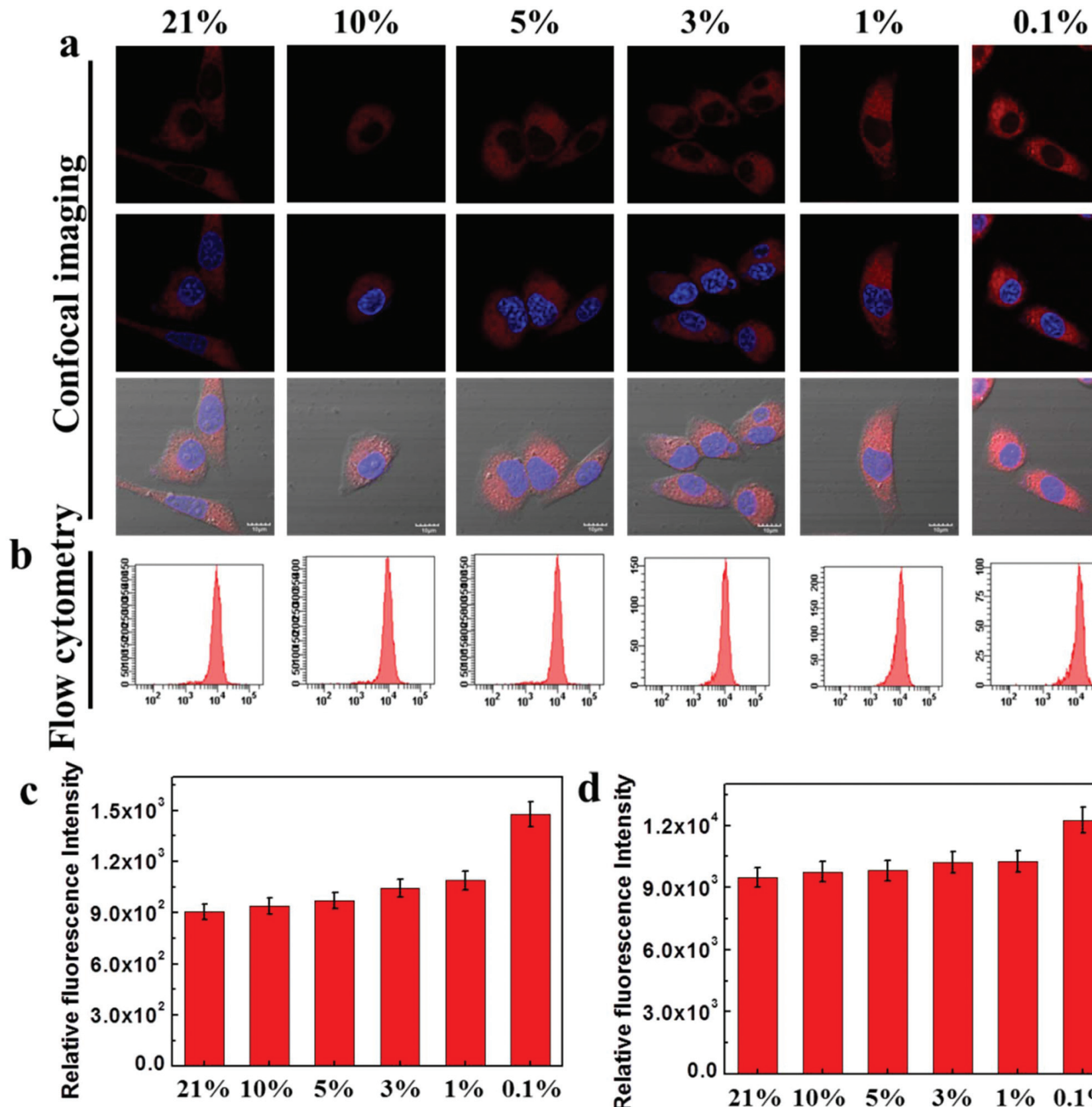
increased with decreasing oxygen concentration. These results were consistent with the results obtained using a laser scanning confocal microscope. The corresponding quantitative analysis of fluorescence of Fig. $2 \mathrm{~b}$ is shown in Fig. $2 \mathrm{~d}$.

\subsection{NTR detection using a two-photon confocal microscope}

Benefiting from the advantages of two-photon fluorescent probes such as longer observation time, higher spatial and temporal resolution and deeper tissue imaging depth, we further performed NTR detection by using a two-photon confocal microscope. The cells, as shown in Fig. 3, were cultured at different oxygen concentrations $(21 \%$ and $0.1 \%)$ for $6 \mathrm{~h}$. Then, the cells were incubated with hTP-NNO $\mathrm{NN}_{2}(10 \mu \mathrm{M})$ for 25 min for NTR detection. As shown in Fig. 3a, we can observe weak fluorescence signals under normoxic conditions (the control group). The cells incubated with $0.1 \%$ oxygen concentration (the hypoxia group) showed strong fluorescence signals (Fig. 3b). We could capture fluorescence signals at each layer throughout the cell to observe their cellular activities. Thus, our probe is a promising tool for NTR detection and observing cellular activities at each layer of deep tissues using a twophoton confocal microscope.

\subsection{NTR detection in deep brain tissue}

After the NTR detection using a two-photon confocal microscope, we further performed NTR detection in deep brain tissue. We selected the brain as the model organ for NTR detection because brain tissue is extremely sensitive to hypoxia. ${ }^{29}$ The hypoxia mouse models were raised in normobaric hypoxic (FIO2 11\%) chambers for 1 day, and the results are shown in Fig. 4. The brain tissue sections were incubated with probe $(10 \mu \mathrm{M})$ for $30 \mathrm{~min}$. The mice in the control group were placed under normoxic conditions, and we could capture weak fluorescence signals (Fig. 4a). The mice that were placed under hypoxia conditions exhibited bright fluorescence signals (Fig. 4b). These results indicated that the NTR level under hypoxia conditions was much higher than that under normoxic conditions. Moreover, the imaging depth of hTP- $\mathrm{NO}_{2}$ can be as high as $100 \mu \mathrm{m}$, which made it possible to capture more detailed information in different tissue depths. We further performed H\&E staining to evaluate the damage in brain tissue. As shown in Fig. 4c and d, the brain tissues in the control group were tightly arranged and the nuclei of the brain cells were regular. However, the brain tissues under hypoxia conditions were disordered and their shape was deformed. These results indicated that hTP- $\mathrm{NNO}_{2}$ can capture more detailed information in deep brain tissue.

\subsection{NTR detection in vivo}

The probe with NIR fluorescence emission can achieve NTR detection with minimal interferences from background autofluorescence in biological systems, minimal photodamage to

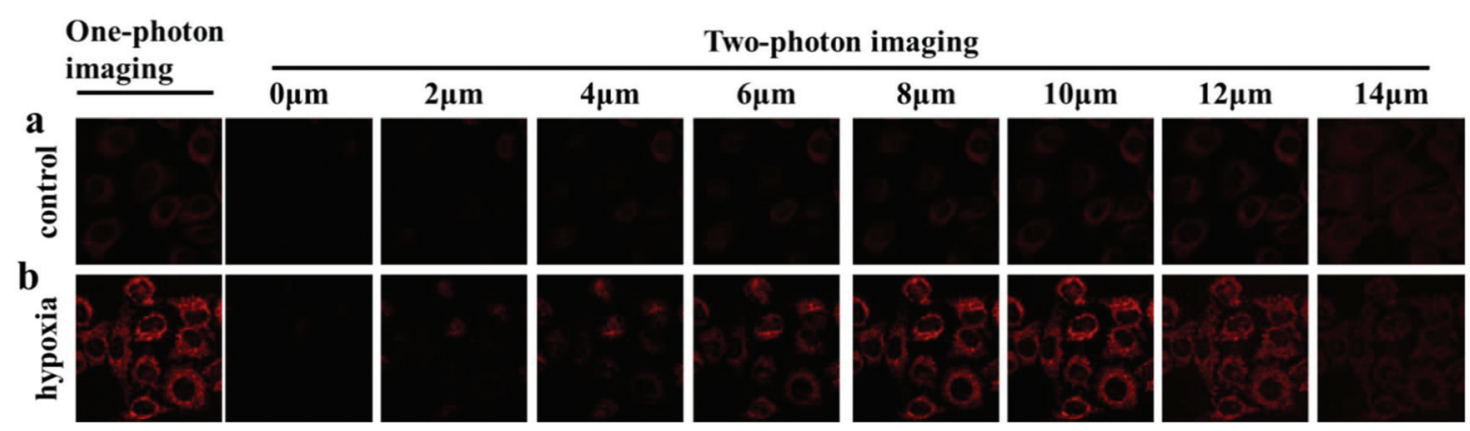

Fig. 3 NTR detection using a one-photon confocal microscope and a two-photon confocal microscope. (a) NTR detection under normal oxygen conditions (21\%). (b) NTR detection under hypoxia conditions (0.1\%). Cells were treated with $10 \mu \mathrm{l}$ of the probe for $25 \mathrm{~min}$ at $37^{\circ} \mathrm{C}$. We used a twophoton confocal microscope to capture fluorescence signals at different imaging depths $(0,2,4,6,8,10,12$, and $14 \mu \mathrm{m})$.

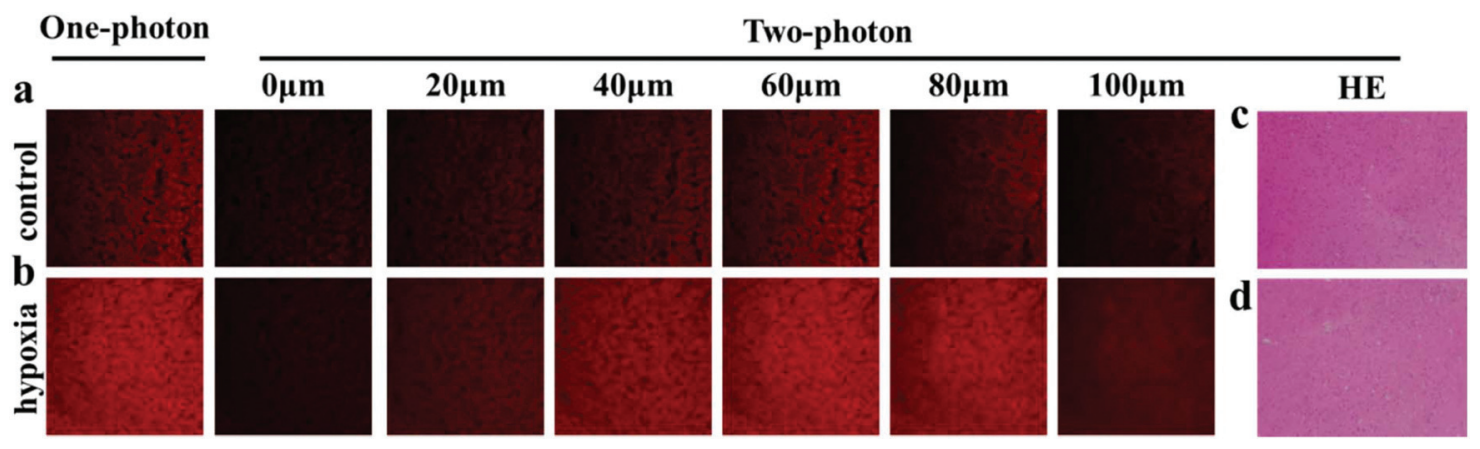

Fig. 4 NTR detection using a confocal microscope under controlled (a) and hypoxia conditions (b). (c) and (d) are the corresponding HE staining images of $a$ and $b$. 
biological samples and deep tissue penetration. It is indispensable to assess the applicability of hTP- $\mathrm{NNO}_{2}$ for NTR detection in vivo. We firstly performed NTR detection in zebrafish, and the results are shown in Fig. 5a. Zebrafish were incubated with different concentrations of oxygen from $21 \%$ to $1 \%$ for $4 \mathrm{~h}$, and then they were treated with hTP-NNO $2(10 \mu \mathrm{M})$ for $20 \mathrm{~min}$. We can observe that the fluorescence intensities increased with a decrease in oxygen concentrations, and the quantitative analysis of fluorescence is shown in Fig. 5d. As is well known, NTR is overexpressed in tumors, and therefore we further performed NTR detection in A549-bearing mice. 5 -Week-old nude mice were injected with $2 \times 10^{6}$ cells subcutaneously, and the A549 xenografts were established in nude mice until the tumor volumes typically reached about $200 \mathrm{~mm}^{3}$. The A549 xenografts of nude mice were intratumorally injected with $\mathrm{hTP}-\mathrm{NNO}_{2}(1 \mu \mathrm{M}, 50 \mu \mathrm{L}$ in 1:99 DMSO/ saline $\mathrm{v} / \mathrm{v})$. The fluorescence signals were collected using a Bruker In vivo Imaging System. As shown in Fig. 5b, the fluorescence signals increased with time, without fluorescence leakage in $30 \mathrm{~min}$. The corresponding quantitative analysis of
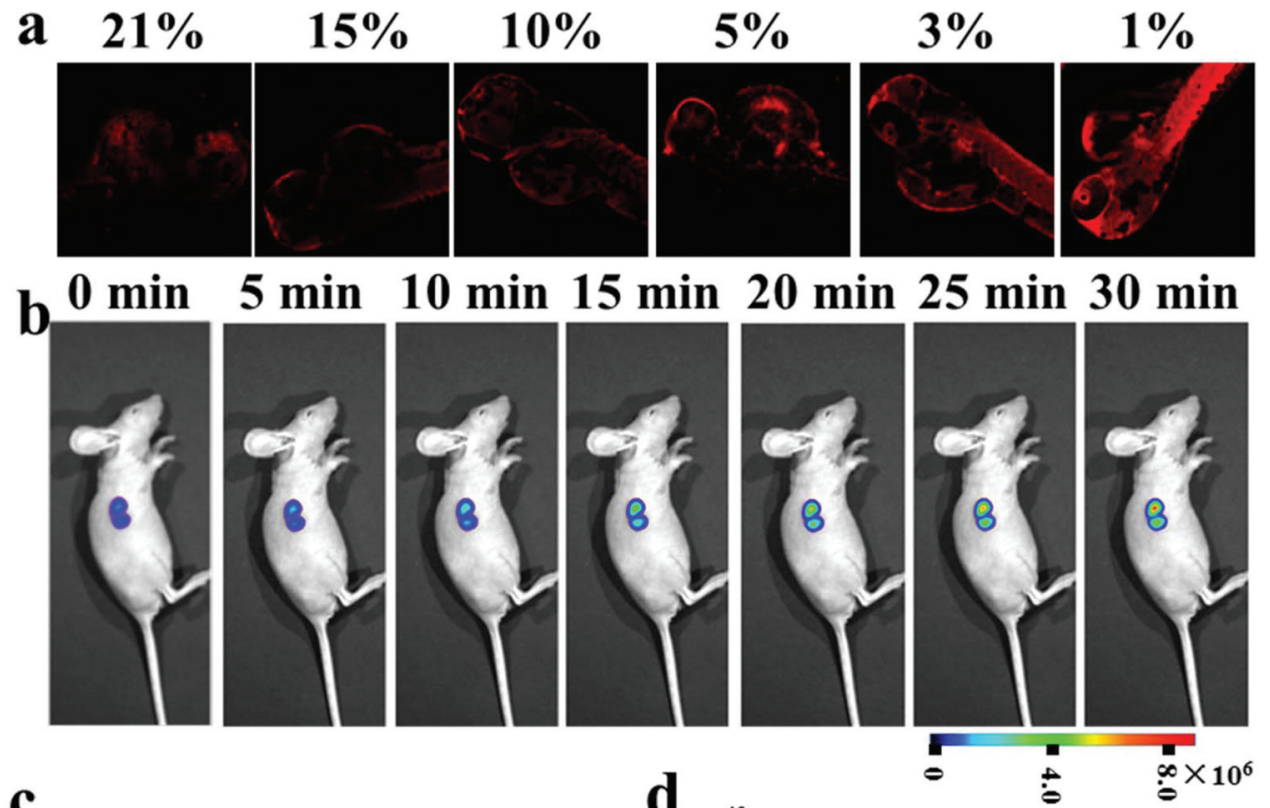

c
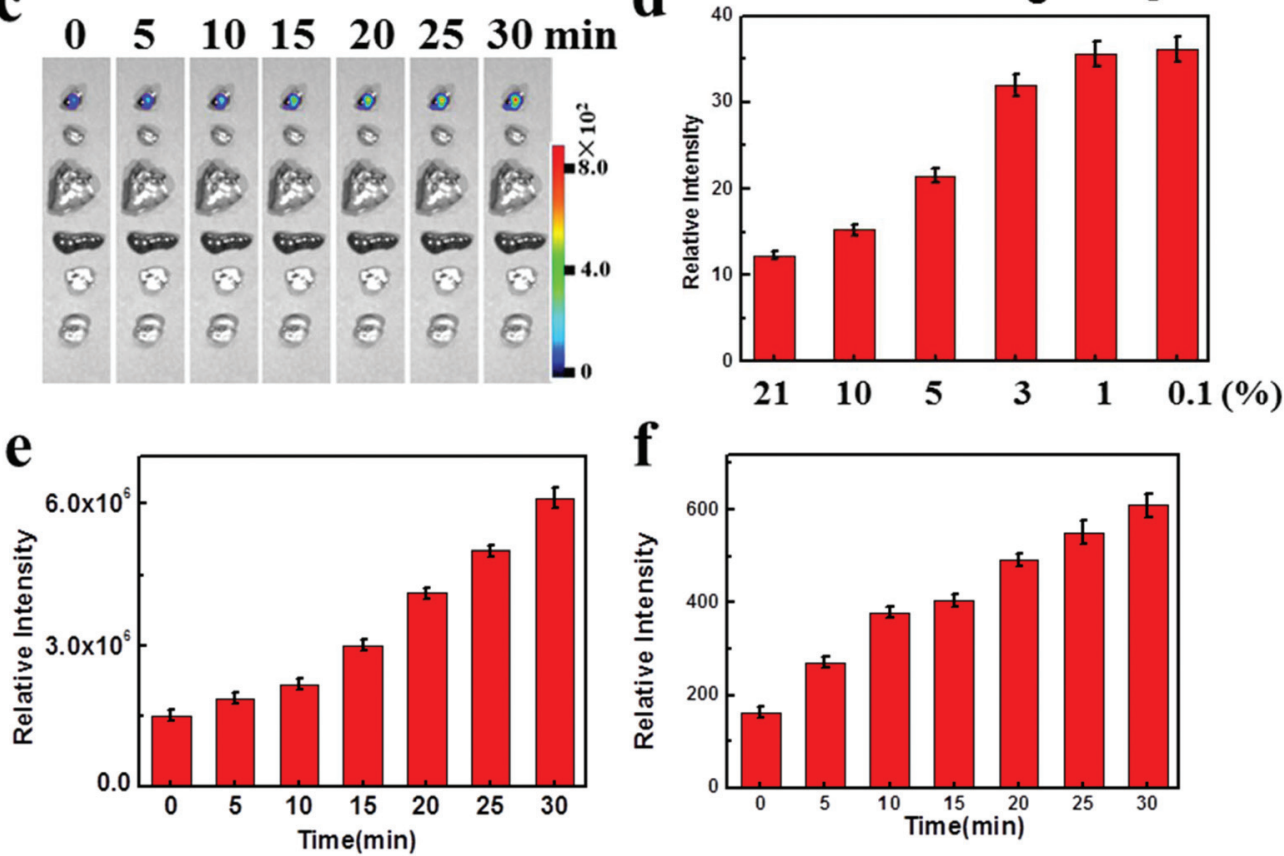

Fig. 5 NTR detection in vivo. (a) NTR detection in zebrafish. (b) A549 tumor-bearing mice were intratumorally injected with hTP-NNO $2(1 \mu M, 50 \mu \mathrm{L}$ in 1: $99 \mathrm{DMSO} / \mathrm{saline} \mathrm{v} / \mathrm{v}$ ) for in vivo NTR detection for 0-30 min. (c) Sub-distribution fluorescence imaging in the heart, liver, spleen, lungs, kidneys and tumors. (d) Corresponding quantitative analysis of fluorescence of a. (e) Corresponding quantitative analysis of fluorescence of b. (f) Corresponding quantitative analysis of fluorescence of $c$. 
fluorescence is shown in Fig. 5e. In addition, the organs, including the heart, liver, spleen, lungs, and kidneys, and tumors were isolated for fluorescence imaging, and the results are shown in Fig. 5c. We can observe fluorescence signals only in the tumors without leakage to their adjacent organs. Thus, hTP-NNO $\mathrm{N}_{2}$ is a promising tool for NTR detection in tumors.

\section{Conclusions}

In summary, we have developed a two-photon fluorescent probe hTP- $\mathrm{NNO}_{2}$ for NTR detection in vitro and in vivo. The probe showed fast response, superior sensitivity and excellent selectivity for NTR detection in complex biological samples. The probe hTP-NNO $\mathrm{N}_{2}$ was successfully applied for NTR detection under hypoxia conditions in zebrafish and A549 xenografts of nude mice without fluorescence leakage to their adjacent organs. Furthermore, the two-photon fluorescent probe was suitable for NTR detection in deep brain tissue and the imaging depth of hTP-NNO $\mathrm{N}_{2}$ was as high as $100 \mu \mathrm{m}$. We anticipate that the probe hTP- $\mathrm{NNO}_{2}$ can be used as a valuable analytical tool for investigating NTR-related physiological and pathological processes in tumors.

\section{Ethics statement}

All surgical procedures were conducted in conformity with the Care and Use of National Guidelines for the laboratory animals, and experimental protocols were approved by the Institutional Animal Care and Use Committee of Binzhou Medical University, Yantai, China. Approval Number: BZ2014-102R.

\section{Conflicts of interest}

There are no conflicts to declare.

\section{Acknowledgements}

We thank the National Nature Science Foundation of China (No. 21976209, 21575159, and 21778026) and the Taishan Scholar Project special funding.

\section{References}

1 J. Zheng, Y. Shen, Z. Xu, Z. Yuan, Y. He, C. Wei and H. Chen, Biosens. Bioelectron., 2018, 119, 141-148.

2 E. McCormack, E. Silden, R. M. West, T. Pavlin, D. R. Micklem, J. B. Lorens and B. T. Gjertsen, Cancer Res., 2013, 73, 1276-1286.

3 K. H. Gebremedhin, Y. Li, Q. Yao, M. Xiao, F. Gao, J. Fan and X. Peng, J. Mater. Chem. B, 2019, 7, 408-414.

4 J. E. Park, H. S. Tan, A. Datta, R. C. Lai, H. Zhang, W. Meng and S. K. Sze, Mol. Cell. Proteomics, 2010, 9, 1085-1099.
5 P. Silva, P. Mendoza, S. Rivas, J. Díaz, C. Moraga, A. F. Quest and V. A. Torres, Oncotarget, 2016, 7, 29548.

6 S. Kizaka-Kondoh and H. Konse-Nagasawa, Cancer Sci., 2009, 100, 1366-1373.

7 K. Bartels, A. Grenz and H. K. Eltzschig, Proc. Natl. Acad. Sci. U. S. A., 2013, 110, 18351-18352.

8 Z. R. Liu, Y. Tang, A. Xu and W. Lin, Biosens. Bioelectron., 2017, 89, 853-858.

9 J. M. Brown and W. R. Wilson, Nat. Rev. Cancer, 2004, 4, 437.

10 B. A. Teicher, Cancer Metastasis Rev., 1994, 13, 139-168.

11 D. R. Fels and C. Koumenis, Cancer Boil. Ther., 2006, 5, 723-728.

12 L. Cui, Y. Zhong, W. Zhu, Y. Xu, Q. Du, X. Wang and Y. Xiao, Org. Lett., 2011, 13, 928-931.

13 Z. Li, X. Li, X. Gao, Y. Zhang, W. Shi and H. Ma, Anal. Chem., 2013, 85, 3926-3932.

14 K. Xu, F. Wang, X. Pan, R. Liu, J. Ma, F. Kong and B. Tang, Chem. Commun., 2013, 49, 2554-2556.

15 J. Zhang, H. W. Liu, X. X. Hu, J. Li, L. H. Liang, X. B. Zhang and W. Tan, Anal. Chem., 2015, 87, 11832-11839.

16 Y. Li, Y. Sun, J. Li, Q. Su, W. Yuan, Y. Dai and F. Li, J. Am. Chem. Soc., 2015, 137, 6407-6416.

17 H. Yasui, T. Kawai, S. Matsumoto, K. Saito, N. Devasahayam, J. B. Mitchell, K. Camphausen, O. Inanami and M. C. Krishna, Free Radical Res., 2017, 51, 861-871.

18 S. Welz, D. Mönnich, C. Pfannenberg, K. Nikolaou, M. Reimold, C. La Fougère, G. Reischl, P. S. Mauz, F. Paulsen, M. Alber, C. Belka, D. Zips and D. Thorwarth, Radiother. Oncol., 2017, 124, 526-532.

19 D. Zhao, L. Jiang and R. P. Mason, Methods Enzymol., 2004, 386, 378-418.

20 Y. Wang, M. Gao, Q. Chen, F. Yu, G. Jiang and L. Chen, Anal. Chem., 2018, 90, 9769-9778.

21 Y. Wang, L. Zhang, S. Zhang, Z. Liu and L. Chen, Anal. Chem., 2019, 91, 7812-7818.

22 J. Cao, J. Campbell, L. Liu, R. P. Mason and A. R. Lippert, Anal. Chem., 2016, 88, 4995-5002.

23 P. Feng, H. Zhang, Q. Deng, W. Liu, L. Yang, G. Li and M. Li, Anal. Chem., 2016, 88, 5610-5614.

24 A. Chevalier, Y. Zhang, O. M. Khdour, J. B. Kaye and S. M. Hecht, J. Am. Chem. Soc., 2016, 138, 12009-12012.

25 J. Zhou, W. Shi, L. H. Li, Q. Y. Gong, X. F. Wu, X. H. Li and H. M. Ma, Chem. - Asian J., 2016, 11, 2719-2724.

26 H. M. Kim and B. R. Cho, Chem. Rev., 2015, 115, 50145055.

27 M. Pawlicki, H. A. Collins, R. G. Denning and H. L. Anderson, Angew. Chem., Int. Ed., 2009, 48, 3244-3266.

28 L. Cui, Y. Zhong, W. Zhu, Y. Xu, Q. Du, X. Wang and Y. Xiao, Org. Lett., 2011, 13, 928-931.

29 M. Erecińska and I. A. Silver, Respir. Physiol., 2001, 128, 263-276.

30 Y. Fang, W. Shi, Y. Hu, X. Li and H. Ma, Chem. Commun., 2018, 54, 5454-5457.

31 Y. Liu, W. Liu, H. Li, W. Yan, X. Yang, D. Liu and J. Zhang, Anal. Chim. Acta, 2018, 1024, 177-186. 